\title{
ROLAND GERBER
}

GOTT IST BURGER ZU BERN

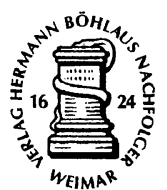


FORSCHUNGEN ZUR MITTELALTERLICHEN GESCHICHTE

Begründet durch Heinrich Sproemberg

Herausgegeben von

Johannes Fried, Peter Moraw und

Eckhard Müller-Mertens

BAND 39 


\author{
Roland Gerber
}

\title{
GOTT IST BURGER ZU BERN
}

\author{
Eine spätmittelalterliche Stadtgesellschaft \\ zwischen Herrschaftsbildung und sozialem Ausgleich
}


Die Deutsche Bibliothek - CIP-Einheitsaufnahme

Gerber, Roland:

Gott ist Burger zu Bern : eine spätmittelalterliche Stadtgesellschaft zwischen Herrschaftsbildung und sozialem Ausgleich / Roland Gerber. - Weimar : Verlag Hermann Böhlaus Nachf., 2001

(Forschungen zur mittelalterlichen Geschichte ; Bd. 39)

ISBN 978-3-7400-1163-5

ISBN 978-3-7400-1163-5

ISBN 978-3-476-02827-3 (eBook)

DOI 10.1007/978-3-476-02827-3

ISSN 0071-7673

Alle Rechte vorbehalten. Ohne schriftliche Genehmigung des Verlages ist es nicht gestattet, das Werk unter Verwendung mechanischer, elektronischer und anderer Systeme in irgendeiner Weise zu verarbeiten und zu verbreiten. Insbesondere vorbehalten sind die Rechte der Vervielfältigung - auch von Teilen des Werkes - auf photomechanischem oder ähnlichem Wege, der tontechnischen Wiedergabe, des Vortrags, der Funk- und Fernsehsendung, der Speicherung in Datenverarbeitungsanlagen, der Übersetzung und der literarischen oder anderweitigen Bearbeitung.

(C) 2001 Springer-Verlag GmbH Deutschland

Ursprünglich erschienen bei Verlag Hermann Böhlaus Nachfolger Weimar GmbH \& Co. 2001

www.boehlausnf.de

info@boehlausnf.de 


\section{Inhaltsverzeichnis}

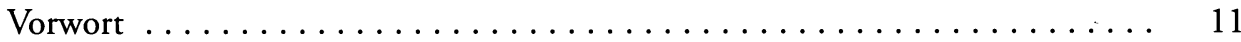

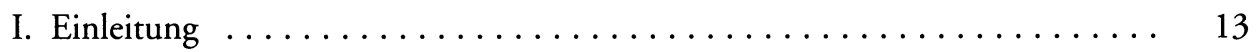

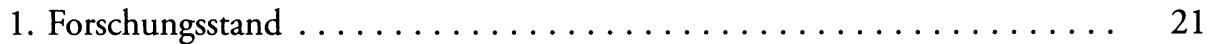

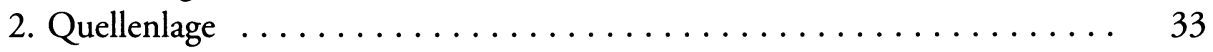

2.1. Die Udelbücher ........................ 33

2.1.1. Das Udelbuch von $1389 \ldots \ldots \ldots \ldots$. . . . . . . . . 34

Anlage und Gliederung

Inhalt und Zweck

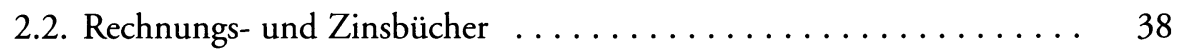

2.3. Übrige Quellen . . . . . . . . . . . . . . . . . . . . . 41

3. Die Verfassungsgrundlagen Berns im Spätmittelalter . . . . . . . . . . 42

3.1. Die Verfassungsreform von $1294 \ldots \ldots \ldots \ldots \ldots \ldots$

3.2. Ratsämter und Behörden ...................... 47

3.2.1. Schultheiss und Rat . . . . . . . . . . . . . . 48

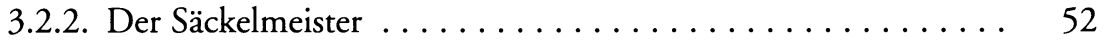

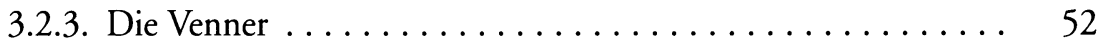

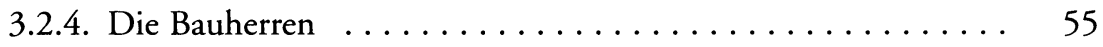

3.2.5. Der Stadtschreiber . . . . . . . . . . . . . . 57

3.2.6. Übrige Ämter und Behörden . . . . . . . . . . . . . . . . . . 58

4. Währungs- und Preisverhältnisse ................. 60

4.1. Der Kampf gegen Inflation und Teuerung $\ldots \ldots \ldots \ldots \ldots \ldots$

4.2. Die Kaufkraft des Geldes ...................... 64

II. Die Stadtbevölkerung $\ldots \ldots \ldots \ldots \ldots \ldots \ldots \ldots \ldots \ldots \ldots$

1. Die Bevölkerungszahlen $\ldots \ldots \ldots \ldots \ldots \ldots \ldots \ldots \ldots \ldots \ldots \ldots$

1.1. Die statistischen Auswertungen $\ldots \ldots \ldots \ldots \ldots \ldots \ldots \ldots \ldots$

1.1.1. Haushaltgrösse und Familie . . . . . . . . . . . . 76

2. Die Bevölkerungs- und Stadtentwicklung . . . . . . . . . . . 79

2.1. Das Wachstum im 13. und 14. Jahrhundert ............ 80 
2.1.1. Die erste Stadterweiterung . . . . . . . . . . . . . . 81

2.1.2. Ausbau und innere Verdichtung $\ldots \ldots \ldots \ldots \ldots \ldots \ldots . \ldots \ldots$

2.1.3. Die zweite Stadterweiterung ................. 88

2.2. Rückgang und Stagnation im 15. Jahrhundert $\ldots \ldots \ldots \ldots \ldots . \quad 89$

2.2.1. Die Pest ............................ 90

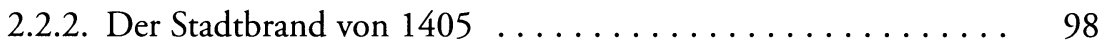

2.2.3. Die Abschliessungstendenzen von Zünften und Bürgerschaft ....................... 101

Die Niederlassungspolitik des Rates

Die Schliessung des Bürgerrechts

2.2.4. Die Verhältnisse auf dem Land ... . . . . . . . . . . . . . . 109

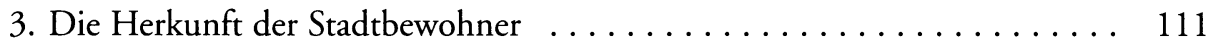

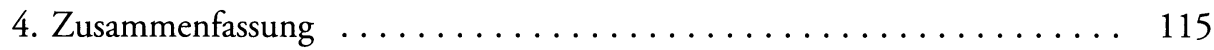

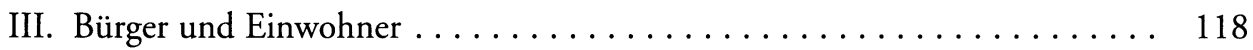

1. Das Bürgerrecht ............................ 119

1.1. Die Aufnahme ins Bürgerrecht ................. 120

1.1.1. Der Bürgereid ....................... 121

Der Neubürger- und Untertaneneid

1.1.2. Das Udel ............................. 127

Der Udelbesitz der Ausbürger

Die haushäblichen Ratsbürger

1.1.3. Die Bürgerpflichten ..................... 133

Die Steuerpflicht

Die Wach- und Wehrpflicht

1.1.4. Die Bürgerrechtsgebühren ................... 139

Udelzins und Burgermäss

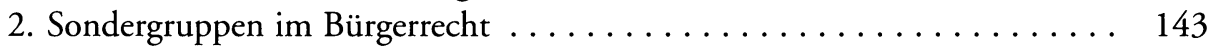

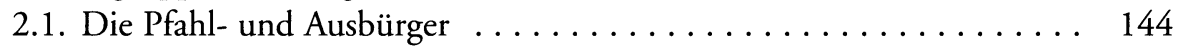

2.2. Die Gedingbürger ...................... 150

2.2.1. Adlige und geistliche Gerichtsherren . . . . . . . . . . 151

2.2.2. Kaufleute, Juden und Lombarden $\ldots \ldots \ldots \ldots \ldots \ldots \ldots . . \ldots 160$

2.3. Die Twingherren ........................ 173

2.4. Die Bürgerinnen ........................ 177

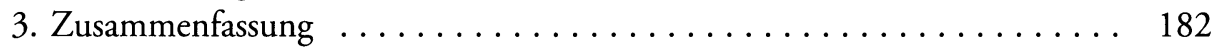

IV. Die räumliche Gliederung des Stadtgebietes . . . . . . . . . . . 185

1. Die Stadtviertel ............................. 186

2. Die Ober- und Unterstadt ...................... 188 
3. Die Kirchgemeinde von St. Vinzenz . . . . . . . . . . . . . . . . 190

4. Die Stadtquartiere . . . . . . . . . . . . . . . . . . . . . 193

4.1. Die Zähringerstadt . . . . . . . . . . . . . . . . . . 194

4.2. Nydeggstalden und Matte . . . . . . . . . . . . . . . . . . . 195

4.2.1. Der Stalden ....................... 196

4.2.2. Die Matte . . . . . . . . . . . . . . . . . . . . . . . . . 197

4.3. Die Innere Neustadt . . . . . . . . . . . . . . . . . . . . . . . 199

4.4. Die Äussere Neustadt . . . . . . . . . . . . . . . . . . . . 200

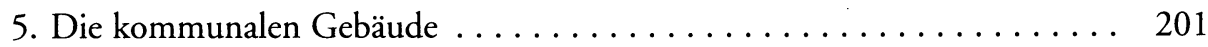

5.1. Schultheissensitz und Rathaus .................. 202

5.2. Die Stadtschule . . . . . . . . . . . . . . . . . . . . . . . . . . 204

5.3. Das Trämmelhaus ....................... 204

5.4. Das Nachrichter- und Frauenhaus . . . . . . . . . . . . . 205

6. Die Zunft- und Gewerbebauten . . . . . . . . . . . . . . . 206

6.1. Die Gewerbehäuser der Bäcker, Metzger und Gerber . . . . . . . . . 206

6.2. Das städtische Kauf- und Zollhaus . . . . . . . . . . . . . . 208

6.3. Die Gesellschaftshäuser . . . . . . . . . . . . . . . . . . . . . . . 209

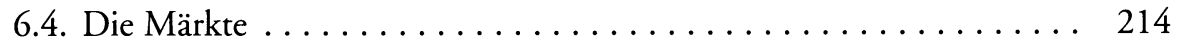

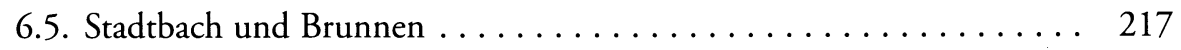

7. Die geistlichen Niederlassungen $\ldots \ldots \ldots \ldots \ldots \ldots \ldots \ldots \ldots \ldots \ldots$

7.1. Die Pfarrkirche St. Vinzenz und das

Franziskanerkloster ...................... 220

7.2. Das Dominikaner- und Inselkloster . . . . . . . . . . . . 221

7.3. Die Nydeggkapelle . . . . . . . . . . . . . . . . . . 222

7.4. Die Spitäler . . . . . . . . . . . . . . . . . . . . . 223

7.4.1. Das Seilerinspital . . . . . . . . . . . . . . . . . 224

7.4.2. Die Elenden Herberge . . . . . . . . . . . . . . . . . . . . . . . . . . 224

7.4.3. Das Antonierspital ................... 225

7.4.4. Das Obere Spital . . . . . . . . . . . . . . . . . 226

7.4.5. Das Niedere Spital .................... 226

7.4.6. Das Siechenhaus ................... 227

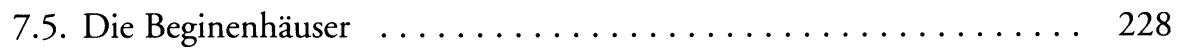

7.5.1. Die Weissen Schwestern . . . . . . . . . . . . . . . . 229

7.5.2. Die Grauen Schwestern . . . . . . . . . . . . . . . 230

7.6. Die Stadthäuser auswärtiger Klöster . . . . . . . . . . . . 233

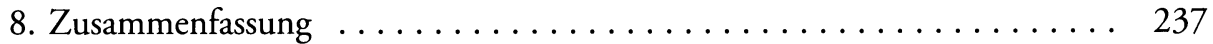

V. Reichtum und politische Macht $\ldots \ldots \ldots \ldots \ldots \ldots \ldots \ldots \ldots \ldots$

1. Die Schuldenpolitik des Rates im 14. Jahrhundert . . . . . . . . . . 240

1.1. Die sozialen Auswirkungen ................... 245 
1.1.1. Die Auseinandersetzungen um die Besetzung des Schultheissenamtes . . . . . . . . . . . . . 246 Die Amtsenthebung Johannes von Bubenbergs Die Rückkehr der Familie von Bubenberg

1.1.2. Innerstädtische Unruhen

Der Geltenhalsaufstand

Die Ratsentsetzung von 1384

2. Die Sanierung des kommunalen Finanzhaushalts im 15. Jahrhundert . . . 262

2.1. Die Ausdehnung der Steuerhoheit auf die Landschaft . . . . . . . . . 263

2.2. Die Einführung der einjährigen Amtszeit der Schultheisse . . . . . . . 265

3. Die Vermögensstruktur der Stadtbevölkerung . . . . . . . . . . . . . 268

3.1. Die Telle von $1389 \ldots \ldots \ldots \ldots$. . . . . . . . . . . . . 270

3.1.1. Die Adels- und Notabelnfamilien . . . . . . . . . . . . . . 273

3.1.2. Die Vermögenstopografie . . . . . . . . . . . . . . . . 277

3.2. Die Telle von $1448 \ldots \ldots \ldots \ldots \ldots \ldots \ldots \ldots \ldots \ldots \ldots$

3.2.1. Die Vermögensentwicklung seit $1389 \ldots \ldots \ldots \ldots . \ldots \ldots$

3.2.2. Die wirtschaftlichen Auf- und Absteiger . . . . . . . . . . 285

3.2.3. Die Twingherrenfamilien . . . . . . . . . . . . . . . 292

3.2.4. Die reichen Witwen . . . . . . . . . . . . . . . 295

3.2.5. Die Zunftbürger . . . . . . . . . . . . . . . . . . . . 298

3.2.6. Die Kopfsteuerzahler . . . . . . . . . . . . . . . . . . . 299

3.2.7. Die soziale Aufwertung von Kram- und Marktgasse . . . . . 301

4. Die reichen Ratsherren . . . . . . . . . . . . . . . . . . 305

4.1. Die Vermögensverhältnisse der Klein- und Grossräte . . . . . . . . . 306

4.1.1. Die Ratslisten von 1383 und $1448 \ldots \ldots \ldots . \ldots . \ldots 308$

4.1.2. Die Wohnlagen der Ratsmitglieder . . . . . . . . . . . 312

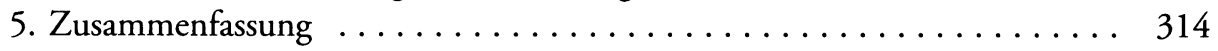

VI. Handel und Gewerbe . . . . . . . . . . . . . . . . . 317

1. Markt und Münze ....................... 318

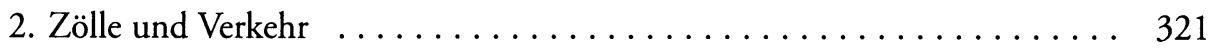

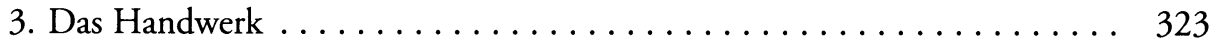

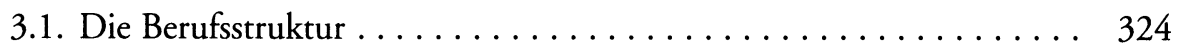

3.2. Die Gewerbeaufsicht des Rates . . . . . . . . . . . . . . . . . . 332

3.2.1. Die Entstehung spezieller Gewerbebezirke . . . . . . . . 334

3.3. Die Berufstopografie ................... 336

3.3.1. Metzger, leder- und fellverarbeitende Berufe . . . . . . . . 337

3.3.2. Metallverarbeitende Berufe ................. 340

3.3.3. Lebensmittelberufe $\ldots \ldots \ldots \ldots \ldots \ldots \ldots \ldots \ldots \ldots \ldots$

3.3.4. Textilberufe ..................... 343 
4. Zünfte und Gesellschaften $\ldots \ldots \ldots \ldots \ldots \ldots \ldots \ldots \ldots$

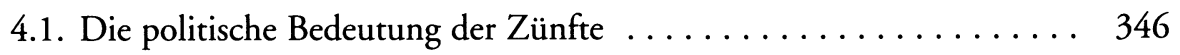

4.1.1. Vennergesellschaften und Vennerviertel . . . . . . . . . 348

4.2. Die Vermögensstruktur von Handwerkerschaft und Zünften . . . . . . 352

4.2.1. Die adlige Stube zum Narren und Distelzwang . . . . . . . . . 355

4.2.2. Die Gesellschaft zum Mittellöwen . . . . . . . . . . . . 359

4.2.3. Die Kaufleutegesellschaft . . . . . . . . . . . . . . 362

4.2.4. Die Vennergesellschaften ................ 366

4.2.5. Die Handwerksgesellschaften . . . . . . . . . . . . 370

5. Zusammenfassung $\ldots \ldots \ldots \ldots \ldots \ldots \ldots \ldots \ldots \ldots \ldots \ldots \ldots$

VII. Das städtische Territorium . . . . . . . . . . . . . . . . . . 377

1. Die Entstehung des städtischen Territoriums . . . . . . . . . 378

1.1. Die Herrschaftsbildung auf dem Land von 1298 bis 1415

1.1.1. Der Burgdorfer- und Sempacherkrieg von 1383 bis $1388 \ldots \ldots \ldots \ldots \ldots \ldots \ldots \ldots \ldots$

1.1.2. Die Übernahme der landgräflichen Rechte durch Bern . . . . . 385

1.1.3. Die Eroberung des Aargaus 1415 . . . . . . . . . . . . 387

1.2. Die Konsolidierung des städtischen Herrschaftsgebietes

im 15. Jahrhundert . . . . . . . . . . . . . . . . . 390

1.2.1. Die Steuer- und Wehrhoheit auf dem Land . . . . . . . . . . 392

1.2.2. Territoriumsgrenzen und Ausbürgerbezirke . . . . . . . . . . 397

Die Stadt Burgdorf

2. Ausbürgeraufnahmen und Udelliegenschaften . . . . . . . . . . 402

2.1. Die politische Bedeutung der Ausbürgeraufnahmen . . . . . . . . 404

2.2. Die sozialen Beziehungen zwischen Ausbürgern

und Stadtbewohnern . . . . . . . . . . . . . . 408

2.2.1. Die Verteilung der Udelhäuser innerhalb des

Stadtgebietes .................... 411

2.2.2. Die Udelhäuser der Adels- und Notabelnfamilien . . . . . . . . 414

2.3. Die veränderte Ausbürgerpolitik im 15. Jahrhundert . . . . . . . . . 418

3. Die städtische Verwaltungsorganisation auf dem Land . . . . . . . . 420

3.1. Die wirtschaftliche Bedeutung der Landvogteien und

die soziale Stellung ihrer Vorsteher . . . . . . . . . . . . . 423

3.1.1. Das Oberland ..................... 425

Thun

Ober- und Niedersimmental

Frutigen

Unterseen und Oberhasli 
3.1.2. Das Seeland ... . . . . . . . . . . . . . . . . . 433

Nidau

Laupen

Büren an der Aare

Aarberg

Oltigen

3.1.3. Das Emmental

Burgdorf

Trachselwald und Brandis

3.1.4. Der Oberaargau

Bechburg und Bipp

Aarwangen

Wangen an der Aare

3.1.5. Der Aargau . . . . . . . . . . . . . . . . . . . . . . . . . . . 459

Lenzburg

Aarburg

4. Zusammenfassung

VIII. Herrschaftsbildung und sozialer Ausgleich

Anhang

1. Abkürzungen . . . . . . . . . . . . . . . . . . . . . . . . . 479

2. Bibliographie ........................ 480

2.1. Quellen .......................... 481

2.1.1. Burgerbibliothek Bern

2.1.2. Staatsarchiv Bern

2.1.3. Stadtarchiv Bern

2.2. Gedruckte Quellen ..................... 481

2.3. Literatur . . . . . . . . . . . . . . . . . . . . . . . 482

3. Verzeichnis der Tabellen . . . . . . . . . . . . . . . . . . . . . 509

4. Personen- und Ortsregister $\ldots \ldots \ldots \ldots \ldots \ldots \ldots \ldots \ldots \ldots \ldots \ldots \ldots$

Abbildungen .......................... 527 


\section{Vorwort}

$\mathrm{Zu}$ Beginn meiner Arbeit über die gesellschaftlichen Verhältnisse in der Stadt Bern während des 14. und 15. Jahrhunderts stand die Herausforderung, die Tausenden in den sogenannten Udelbüchern von 1389 und 1466 überlieferten Namen der steuer- und wehrpflichtigen Berner Bürger auf dem Land und deren Hausbesitz in der Stadt elektronisch zu erfassen. Denn nur auf diese Weise liessen sich die unzähligen sozialgeschichtlichen Informationen in den Udelbüchern - die bereits im 15. Jahrhundert als so unübersichtlich bezeichnet wurden, dass sie der Stadtschreiber kaum mehr lesen konnte - systematisch ordnen und mit Hilfe von Karten und Grafiken auswerten. Auf dem Frontispiz des zweiten Udelbuches findet sich zudem eine für mich richtungsweisende symbolische Darstellung der Herrschaftsauffassung des Berner Rates aus dem Jahre 1466. Die spätgotische Illumination fasst sozusagen die Konzeption meiner Arbeit in bildlicher Form zusammen (s. das Umschlagbild). Indem nämlich der Berner Rat dem auf zwei jungen Bären ruhenden Stadtwappen durch zwei Engel die Krone der römischdeutschen Kaiser aufsetzen liess, versinnbildlichte er in einer für eine spätmittelalterliche Stadt nördlich der Alpen bemerkenswerten Weise seinen von Gott und Kaiser legitimierten Herrschaftsanspruch über das seit dem 14. Jahrhundert erworbene städtische Territorium.

Das Wissen um die Erstellung und die Verwaltung komplexer Personendatenbanken sowie um die visuelle Umsetzung von historischen Erkenntnissen auf Grafiken und Karten erarbeitete ich mir während meiner mehrjährigen Tätigkeit als wissenschaftlicher Mitarbeiter in dem vom Schweizerischen Nationalfonds finanzierten Forschungsprojekt „Neubürger im späten Mittelalter. Migration und Austausch in der Städtelandschaft des alten Reiches 1250 bis 1550 “. Die unermüdliche Förderung durch den Projektleiter und der rege wissenschaftliche Austausch mit meinen Kolleginnen und Kollegen am Historischen Institut der Universität Bern waren denn auch der geistige und materielle Nährboden, auf dem die vorliegende Dissertation überhaupt entstehen konnte. Mein Dank gilt deshalb an erster Stelle meinem Doktorvater Professor Rainer C. Schwinges, an dessen Lehrstuhl ich zwischen 1992 und 1999 angestellt war. Des Weiteren danke ich Dr. Christian Hesse für die unzähligen befruchtenden Gespräche und das Korrekturlesen meines Manuskriptes sowie Dr. Bruno Koch, der mich in Sachen „Datenbank-Design“ und "Software" immer kompetent beraten hat. Tatkräftige Unterstützung in allen Fragen zur bernischen Geschichte fand ich zudem bei Professor Urs Martin Zahnd und Vinzenz Bartlome sowie bei der Herausgeberin der Berner Rechtsquellen auf dem Land 
Dr. Anne-Marie Dubler, die mir ihre Lexikonartikel zur Geschichte der bernischen Gemeinden zur Verfügung gestellt hat. Besonderes Lob verdient schliesslich auch die enge Zusammenarbeit mit den Mitarbeiterinnen und Mitarbeitern des Archäologischen Dienstes des Kantons Bern. Vor allem Armand Baeriswyl danke ich für die zahlreichen interessanten Einblicke in die mittelalterliche Stadtgeschichte Berns unter dem heutigen Strassenniveau.

Ermöglicht wurde die Drucklegung meiner Dissertation durch die finanzielle Unterstützung des Schweizerischen Nationalfonds zur Förderung des wissenschaftlichen Nachwuchses und der Burgergemeinde Bern. Ich danke beiden Institutionen für das Vertrauen, das sie meiner wissenschaftlichen Tätigkeit entgegenbringen. Des Weiteren danke ich den Herren Professoren Johannes Fried, Peter Moraw und Eckhard MüllerMertens, die meine Arbeit als 39sten Band in die Reihe „Forschungen zur mittelalterlichen Geschichte" aufgenommen haben. Besonderen Dank schulde ich nicht zuletzt auch dem Vorstand und den Mitgliedern des Historischen Vereins des Kantons Bern für das Interesse an meinen Forschungen sowie den Mitarbeiterinnen und Mitarbeitern des Berner Staatsarchivs, auf dessen reichem Quellenmaterial das vorliegende Werk zu einem grossen Teil beruht.

Ich widme dieses Buch meiner Ehefrau Eva Schärer. 